P. Lescot

Nagoya Math. J.

Vol. 163 (2001), 71-85

\title{
KERNEL SYSTEMS ON FINITE GROUPS
}

\author{
PAUL LESCOT
}

\begin{abstract}
We introduce a notion of kernel systems on finite groups: roughly speaking, a kernel system on the finite group $G$ consists in the data of a pseudoFrobenius kernel in each maximal solvable subgroup of $G$, subject to certain natural conditions. In particular, each finite $C A$-group can be equipped with a canonical kernel system. We succeed in determining all finite groups with kernel system that also possess a Hall $p^{\prime}$-subgroup for some prime factor $p$ of their order; this generalizes a previous result of ours (Communications in Algebra 18(3), 1990, pp. 833-838). Remarkable is the fact that we make no a priori abelianness hypothesis on the Sylow subgroups.
\end{abstract}

\section{$\S 0$. Introduction}

In this paper, we shall define a new class of finite groups, that contains the class of $C A$-groups, and shall derive $(\S 1)$ its basic properties. Then, $C N^{*}$-groups will be defined via an extra hypothesis, and studied(§2). In the case (§3) that there also exists a solvable $p^{\prime}$-Hall subgroup of the $C N^{*}$ group $G$ (for some prime $p \in \pi(G)$ ), we shall obtain a generalization of the main Theorem of [4].

This work was inspired by the conditions stated in p. ix of [1]. I am also much indebted to John Thompson for many enlightening comments on [4], in particular those contained in [6].

The notations are mostly standard; for $G$ a group and $A \subseteq G$, we denote

$$
A^{\sharp}=A \cap(G \backslash\{1\}) ;
$$

for $(x, y) \in G \times G$

$$
y^{x}=x^{-1} y x ;
$$

and, for $A \subseteq G$ and $x \in G$ :

$$
A^{x}=\left\{y^{x} \mid y \in A\right\}
$$

Received April 10, 2000.

2000 Mathematics Subject Classification: 20E25. 
$\mathcal{M S}(G)$ denotes the set of maximal solvable subgroups of $G$. A finite group $G$ will be termed $C A$ (resp. $C N, C S$ ) if, for each $x \in G^{\sharp}$, the centralizer $C_{G}(x)$ is abelian (resp. nilpotent, solvable).

\section{$\S 1$. Definition and first properties of kernel systems}

Definition 1.1. By a kernel system on the finite group $G$ we shall mean an application

$$
\mathcal{F}: M \mapsto M_{0}=\mathcal{F}(M)
$$

from $\mathcal{M S}(G)$ to $\mathcal{P}(G)$ such that, for each $M \in \mathcal{M S}(G)$ : $M_{0}$ is a normal subgroup of $M$,

$$
\begin{aligned}
& \forall a \in M \backslash M_{0} \quad C_{M_{0}}(a)=\{1\}, \quad \text { and } \\
& \forall g \in G \backslash M \quad M_{0} \cap M_{0}^{g}=\{1\} .
\end{aligned}
$$

On every finite group can be defined a trivial kernel system by:

$$
\forall M \in \mathcal{M S}(G) \quad M_{0}=\{1\} .
$$

More interesting is :

Lemma 1.2. If $G$ is a $C A$-group, then $G$ possesses a canonical kernel system.

Proof. Let $G$ be a $C A$-group; if $G$ is solvable then $\mathcal{M S}(G)=\{G\}$, and (see for example Theorem 1.3 of [4]) $G$ is either abelian or a Frobenius group with an abelian kernel (let it be $A$ ) that is also a maximal abelian subgroup of $G$, and a cyclic complement. In the first case, $G_{0}=G$ is suitable; in the second case, $G_{0}=A$ works, thanks to Lemma 1.2 of [4].

We may therefore assume that $G$ is not solvable; hence it is (nonabelian) simple by the result of [7], p.416. It now follows from Theorem 1.4 of [4] that the elements of $\mathcal{M S}(G)$ are exactly the $N_{G}(A)$, for $A$ a maximal abelian subgroup of $G$; setting $\left(N_{G}(A)\right)_{0}=A$ for all such $A$ yields the result, again thanks to Lemma 1.2 of [4].

By a $K S$-group we shall mean a pair $(G, \mathcal{F})$, with $G$ a finite group and $\mathcal{F}$ a kernel system on $G$. If $\mathcal{F}$ is clear from (or fixed in) the context, we shall term $G$ itself a $K S$-group. In particular, if $G$ is a $C A$-group, it will be considered as a $K S$-group via the canonical kernel system defined in the proof of Lemma 1.2.

In the following three lemmas, let $G$ be a $K S$-group. 
Lemma 1.3. Let $M \in \mathcal{M S}(G)$, and let $x \in M_{0}^{\sharp} ;$ then $C_{G}(x) \subseteq M_{0}$.

Proof. If $a \in C_{G}(x)$, then $1 \neq x=x^{a} \in M_{0} \cap M_{0}^{a}$, whence $a \in M$ by (3). If $a$ would belong to $M \backslash M_{0}$, then (2) would yield $x \in C_{M_{0}}(a)=\{1\}$, a contradiction. Therefore $a \in M_{0}$.

Corollary 1.4. For each $M \in \mathcal{M S}(G), M_{0}$ is a Hall subgroup of $G$ (and hence of $M$ ).

Proof. This follows immediately from Lemma 1.3 by using Lemma 1.1 of $[4]$.

Proposition 1.5. ([1], p.x) If $M \in \mathcal{M S}(G)$ and $M_{0} \neq M$, then $M_{0}$ is nilpotent.

Proof. Assume $M_{0} \neq M$, and let $q \in \pi\left(\frac{M}{M_{0}}\right)$; then Corollary 1.4 yields that $M_{0}$ is a $q^{\prime}$-group. Let $x \in M$ have order $q$; then $x \notin M_{0}$, whence $C_{M_{0}}(x)=\{1\}$ by (2). Therefore $M_{0}$ has a fixed-point-free automorphism of order 1 or $q$ (induced by conjugation by $x$ ), hence is nilpotent by [5], 12.6.13, p.354 (we do not need Thompson's Theorem here because we already know that $M_{0} \subseteq M$ is solvable).

\section{$\S 2 . C N^{*}$-groups}

Definition 2.1. A $K S$-group will be termed a $C N^{*}$-group if it satisfies:

(4) $G=\bigcup_{M \in \mathcal{M S}(G)} M_{0}$, and :

(5) For all $M \in \mathcal{M S}(G), \frac{M}{M_{0}}$ is a nonidentity cyclic group.

Proposition 2.2. ([1], p.x) Let $G$ be a KS-group such that (5) holds and either:

(i) (4) holds (i.e. G is a $C N^{*}$-group)

or

(ii) $G$ is a $C S$-group.

Then $G$ is a $C N$-group. 
Proof. Let $x \in G^{\sharp}$.

In case (i) $x$ belongs to $M_{0}^{\sharp}$ for some $M \in \mathcal{M S}(G)$, by (4). By Lemma 1.3, $C_{G}(x) \subseteq M_{0}$. But $M_{0}$ is nilpotent according to Proposition 1.5 and (5), hence so is $C_{G}(x)$.

In case (ii), $C_{G}(x)$ is solvable, hence $C_{G}(x) \subseteq M$ for some $M \in \mathcal{M S}(G)$. Clearly $x \in M^{\sharp}$; if $x \in M_{0}^{\sharp}$, then $C_{G}(x) \subseteq M_{0}$ is nilpotent, as above. If $x \in M \backslash M_{0}$ then

$$
C_{G}(x) \cap M_{0}=C_{M_{0}}(x)=\{1\}
$$

because of (2), thus $C_{G}(x)$ is isomorphic to a subgroup of $\frac{M}{M_{0}}$, hence is cyclic and a fortiori nilpotent.

Lemma 2.3. Let $G$ be a $C N^{*}$-group, let $q \in \pi(G)$, and let $Q \in \mathcal{S} y l_{q}(G)$; then $N_{G}(Q) \in \mathcal{M S}(G)$ and $Q$ is the unique Sylow q-subgroup of $N_{G}(Q)_{0}$.

Proof. $Q \neq\{1\}$, therefore by (4) one can find $M \in \mathcal{M S}(G)$ such that

$$
Q \cap M_{0} \neq\{1\}
$$

let $x \in Q \cap M_{0}, x \neq 1$. Then, for any $y \in Z(Q)$, one has $1 \neq x=x^{y} \in$ $M_{0} \cap M_{0}^{y}$, whence $y \in M$ by (3), that is $Z(Q) \subseteq Q \cap M$. Let then $u \in Z(Q)$, $u \neq 1$ be fixed; if $u \in M \backslash M_{0}$, then $x \in Q \cap M_{0} \subseteq C_{M_{0}}(u)=\{1\}$, a contradiction. Therefore $u \in M_{0}^{\sharp}$, whence $Q \subseteq C_{G}(u) \subseteq M_{0}$ by Lemma 1.3. Hence $Q$ is a Sylow $q$-subgroup of $M_{0}$; according to Proposition 1.5, $Q=O_{q}\left(M_{0}\right) \triangleleft M$, whence $M \subseteq N_{G}(Q)$.

Let now $y \in N_{G}(Q)$; then $1 \neq Q=Q^{y} \subseteq M_{0} \cap M_{0}^{y}$, whence $y \in M$ by $(3)$. Therefore $N_{G}(Q) \subseteq M$, and $N_{G}(Q)=M \in \mathcal{M S}(G)$. The last part of the statement has already been proved.

Proposition 2.4. Let $G$ be a $C N^{*}$-group, and let $M$ and $N$ be two nonconjugate maximal solvable subgroups of $G$; then $\left(\left|M_{0}\right|,\left|N_{0}\right|\right)=1$.

Proof. If not, let $q \in \pi(G)$ divide both $\left|M_{0}\right|$ and $\left|N_{0}\right|$, and let $Q_{1}$ and $Q_{2}$ be Sylow $q$-subgroups of, respectively, $M_{0}$ and $N_{0} . Q_{1}$ is contained in a Sylow $q$-subgroup $Q$ of $G$, and $Q_{2}$ in a conjugate $Q^{x}$ of $Q$; obviously:

$$
\begin{aligned}
& \{1\} \neq Q_{1}=Q \cap M_{0}, \text { and : } \\
& \{1\} \neq Q_{2}=Q^{x} \cap N_{0} .
\end{aligned}
$$

By the reasoning in the proof of Lemma 2.3, $M=N_{G}(Q)$ and $N=N_{G}\left(Q^{x}\right)$, whence $N=M^{x}$. 
Lemma 2.5. Let $G$ be a $C N^{*}$-group, and let $M \in \mathcal{M S}(G)$ with $M_{0} \neq$ 1; then:

(i) $M=N_{G}\left(M_{0}\right)$, and:

(ii) For each $x \in G$ with $\left(M^{x}\right)_{0} \neq\{1\}$, one has:

$$
\left(M^{x}\right)_{0}=M_{0}^{x} .
$$

\section{Proof.}

(i) By (1), $M \subseteq N_{G}\left(M_{0}\right)$; let $g \in N_{G}\left(M_{0}\right)$. Then

$$
\{1\} \neq M_{0}=M_{0}^{g}=M_{0} \cap M_{0}^{g}
$$

whence $g \in M$ by $(3)$ and $N_{G}\left(M_{0}\right) \subseteq M$ : we have shown that $M=$ $N_{G}\left(M_{0}\right)$.

(ii) Let $Q$ be a Sylow $q$-subgroup of $M_{0}^{x}, Q \neq 1$; then, according to Lemma 2.3 and its proof,

$$
M=N_{G}\left(Q^{x^{-1}}\right)=\left(N_{G}(Q)\right)^{x^{-1}} .
$$

If $Q \nsubseteq\left(M^{x}\right)_{0}$, let $u \in Q \backslash\left(M^{x}\right)_{0}$; then:

$$
Z(Q) \cap\left(Q \cap\left(M^{x}\right)_{0}\right)=Z(Q) \cap\left(M^{x}\right)_{0} \subseteq C_{\left(M^{x}\right)_{0}}(u)=\{1\}
$$

by (2). But $Q \cap\left(M^{x}\right)_{0} \triangleleft Q \cap M^{x}=Q$, hence $Q \cap\left(M^{x}\right)_{0}=\{1\}$. Therefore $Q$ and $\left(M^{x}\right)_{0}$ are both, according to $(*)$, normal subgroups of $M^{x}$, thus they centralize one another; let $1 \neq y \in Q$. Then

$$
\left(M^{x}\right)_{0}=C_{\left(M^{x}\right)_{0}}(y)=\{1\}
$$

by (2), a contradiction. Therefore $Q \subseteq\left(M^{x}\right)_{0}$; it follows that $M_{0}^{x} \subseteq$ $\left(M^{x}\right)_{0}$. Applying the same reasoning to $M^{x}$ and $x^{-1}$ in place of $M$ and $x$ yields $\left(\left(M^{x}\right)_{0}\right)^{x^{-1}} \subseteq\left(\left(M^{x}\right)^{x^{-1}}\right)_{0}=M_{0}$, i.e. $\left(M^{x}\right)_{0} \subseteq M_{0}^{x}$ and $\left(M^{x}\right)_{0}=M_{0}^{x}$.

Important is :

Proposition 2.6. Let $G$ be a nonsolvable $C A$-group; then $G$ is a $C N^{*}$-group.

Proof. This follows, again, from Theorem 1.4 in [4]. 


\section{$\S 3$. The factorizability hypothesis and the main theorem}

In this paragraph, we shall assume the following hypothesis:

$(\mathcal{H}) . \quad G$ is a nonsolvable $C N^{*}$-group, $p \in \pi(G)$, and $H$ is a solvable Hall $p^{\prime}$-subgroup of $G$.

Let $P$ be a Sylow $p$-subgroup of $G$, and let $p^{n}=|P|$.

Lemma 3.1. $C_{G}(P)=Z(P)$

Proof. By a well-known consequence of Burnside's p-nilpotence criterion,

$$
C_{G}(P)=Z(P) \times D
$$

where $D$ is a $p^{\prime}$-group. Therefore

$$
P C_{G}(P)=P Z(P) D=P D=P \times D
$$

(because $D \subseteq C_{G}(P)$ ), and

$$
\begin{aligned}
P \times D & =(P \times D) \cap G \\
& =(P \times D) \cap P H \\
& =P[(P \times D) \cap H] \\
& =P(D \cap H) \text { (because } H \text { is a } p^{\prime} \text {-group) } \\
& =P \times(D \cap H)
\end{aligned}
$$

whence $D=D \cap H$ :

$$
D \subseteq H .
$$

The same reasoning applies to each $P^{x}(x \in G), D$ being replaced by $D^{x}$; therefore

$$
N=<D^{x} \mid x \in G>\subseteq H .
$$

Let us assume $D \neq\{1\}$; then $N$ is a nonidentity solvable normal $p^{\prime}$ subgroup of $G$. Let $N_{1}$ be a minimal normal subgroup of $G$ contained in $N$; then $N_{1}$ is an elementary abelian $q$-group for some prime $q \neq p$. Let $Q$ be a Sylow $q$-subgroup of $G$ that contains $N_{1}$; then $Q \subseteq M_{0}$ for some $M \in \mathcal{M S}(G)$, by Lemma 2.3 (in fact $M=N_{G}(Q)$ ). It follows that, for each $x \in G$ :

$$
\{1\} \neq N_{1}=N_{1}^{x} \subseteq Q \cap Q^{x} \subseteq M_{0} \cap M_{0}^{x}
$$

whence $x \in M$. Therefore $G=M$ is solvable, a contradiction. Thus $D=\{1\}$ and $C_{G}(P)=Z(P) \times D=Z(P)$. 
Corollary 3.2. $N_{G}(P) \in \mathcal{M S}(G)$ and $P=N_{G}(P)_{0}$.

Proof. By Lemma 2.3, $N_{G}(P) \in \mathcal{M S}(G)$ and $P$ is the unique Sylow $p$-subgroup of the nilpotent group $\left(N_{G}(P)\right)_{0}$; therefore $P \subseteq N_{G}(P)_{0} \subseteq$ $P C_{G}(P)=P$, whence $P=\left(N_{G}(P)\right)_{0}$.

LEMMA 3.3. $H$ is not nilpotent and $H_{0} \neq\{1\}$.

Proof. If $H$ were nilpotent, $G=P H$ would be the product of two finite nilpotent groups, hence solvable by a result of Kegel ([3],Satz 2), which is not the case. Therefore $H$ is not nilpotent; but $\frac{H}{H_{0}}$ is cyclic, hence nilpotent. Thus $H$ and $\frac{H}{H_{0}}$ are not isomorphic, thence $H_{0} \neq\{1\}$.

Proposition 3.4. $H \in \mathcal{M S}(G) ; H$ and $N_{G}(P)$ are not conjugate in $G$.

Proof. Let $M \in \mathcal{M S}(G)$ contain $H$; if $p$ would divide $\left|M_{0}\right|$, then for some $x \in G$ one would have $P^{x} \cap M_{0} \neq\{1\}$, whence $M=N_{G}\left(P^{x}\right)$ by the proof of Lemma 2.3. But then $M$ would contain $P^{x} H=G$, contradicting the nonsolvability of $G$. Therefore $M_{0}$ is a $p^{\prime}$-group. Let $x \in M$ be such that $x M_{0}$ generate $\frac{M}{M_{0}}$; if $p$ would divide the order of $x$, then some power $x^{k} \neq 1$ of $x$ would be a $p$-element, hence belong to some conjugate $P^{y}$ of $P$, and one would have :

$$
x \in C_{G}\left(x^{k}\right) \subseteq N_{G}\left(P^{y}\right)_{0}=P^{y}
$$

by Lemma 1.3 applied to $x^{k}$ and $N_{G}\left(P^{y}\right)$, and Corollary 3.2 applied to $P^{y}$. Therefore $x$ would be a $p$-element and $\frac{M}{M_{0}}$ a $p$-group. But

$$
\frac{H M_{0}}{M_{0}} \simeq \frac{H}{H \cap M_{0}}
$$

is a $p^{\prime}$-subgroup of $\frac{M}{M_{0}}$, therefore it would be trivial and $H \subseteq M_{0}$ would be nilpotent, in contradiction with Lemma 3.3. We have shown that $x$ is a $p^{\prime}$-element, hence that $\frac{M}{M_{0}}$ is a $p^{\prime}$-group; therefore so is $M$, whence $|M|$ divides $|G|_{p^{\prime}}=|H|$ and

$$
H=M \in \mathcal{M S}(G)
$$


The second assertion is obvious (and has, in fact, been incidentally proved above).

Remark. This reasoning is adapted from the proof of Step 4 of [4] in an unpublished preliminary version of that paper.

Proposition 3.4 and (5) yield that $\frac{H}{H_{0}}$ is cyclic; let $h \in H$ be such that $h H_{0}$ generate $\frac{H}{H_{0}}$. By $(5), h \neq 1 ;(4)$ implies the existence of $N \in \mathcal{M S}(G)$ such that $h \in N_{0}^{\sharp}$.

Lemma 3.5. $N$ is not conjugate to either $H$ or $N_{G}(P)$.

Proof. If $N=N_{G}(P)^{x}=N_{G}\left(P^{x}\right)$, then $N_{0}=P^{x}$ by Corollary 3.2 applied to $P^{x}$, whence $1 \neq h \in P^{x} \cap H$, a patent contradiction. If $N=H^{x}$, then $H_{0} \neq\{1\}$ by Lemma 3.3 and $\left(H^{x}\right)_{0}=N_{0} \ni h \neq 1$, and Lemma 2.5 yields $H_{0}^{x}=\left(H^{x}\right)_{0}=N_{0}$, whence $1 \neq h \in H_{0}^{x}$, i.e. $h^{x^{-1}} \in H_{0}$. Therefore $\omega(h)=\omega\left(h^{x^{-1}}\right)|| H_{0} \mid$, and

$$
\left|\frac{H}{H_{0}}\right|=\omega\left(h H_{0}\right) \mid\left(\left|\frac{H}{H_{0}}\right|,\left|H_{0}\right|\right)
$$

which is 1 by Corollary 1.4, thus $\frac{H}{H_{0}}=\{1\}$, again contradicting Lemma 3.3 .

Proposition 3.6. Let $M \in \mathcal{M S}(G)$ with $M_{0} \neq\{1\}$; then $M$ is conjugate to $N, H$ or $N_{G}(P)$.

Proof. Let $q$ be a prime divisor of $\left|M_{0}\right|$; if $q=p$, then, for some $y \in G, P^{y} \cap M_{0} \neq\{1\}$, and it appears from the proof of Lemma 2.3 that $M=N_{G}\left(P^{y}\right)=\left(N_{G}(P)\right)^{y}$. If $q \neq p$, then

$$
\left.q|| G\right|_{p^{\prime}}=|H|=\left|\frac{H}{H_{0}}\right|\left|H_{0}\right| .
$$

If now $q|| H_{0} \mid$, then $\left(\left|H_{0}\right|,\left|M_{0}\right|\right) \neq 1$, therefore $M$ is conjugate to $H$ by Proposition 2.4. We are left with the case $q|| \frac{H}{H_{0}} \mid$, that is $q \mid \omega\left(h H_{0}\right)$; but then $q|\omega(h)|\left|N_{0}\right|$, whence $\left(\left|N_{0}\right|,\left|M_{0}\right|\right) \neq 1$, and now $M$ is conjugate to $N$. 


\section{Corollary 3.7.}

$$
|G| \leq 1+\left|G: N_{G}(P)\right|(|P|-1)+|G: H|\left(\left|H_{0}\right|-1\right)+|G: N|\left(\left|N_{0}\right|-1\right) .
$$

Proof. By (4), one has

$$
G^{\sharp}=\bigcup_{M \in \mathcal{M S}(G)} M_{0}^{\sharp} ;
$$

if $M \in \mathcal{M S}(G)$ is such that $M_{0}^{\sharp} \neq \emptyset$, then, by Proposition 3.6, $M=A^{x}$ for some $x \in G$ and some $A \in\left\{N_{G}(P), H, N\right\}$. Thus $A_{0} \neq\{1\}$ and $M_{0} \neq\{1\}$; Lemma 2.5 now shows that $M_{0}=A_{0}^{x}$, whence $\left|M_{0}^{\sharp}\right|=\left|A_{0}\right|-1$. But the total number of conjugates of $A_{0}$ is $\left|G: N_{G}\left(A_{0}\right)\right|=|G: A|$, also by Lemma 2.5 .

From now on, we shall follow very closely the reasoning of [4], pages 836-837.

Lemma 3.8. $\left|G: N_{G}(P)\right|=1+\lambda p^{n}$, for some $\lambda \geq 1$.

Proof. Let $Q=P^{y} \neq P$ be a conjugate of $P$, and let $M=N_{G}(P)(\in$ $\mathcal{M S}(G))$. If $P \cap Q \neq\{1\}$ then

$$
\{1\} \neq P \cap P^{y} \subseteq M_{0} \cap M_{0}^{y}
$$

whence, by (3), $y \in M=N_{G}(P)$ and $Q=P^{y}=P$, a contradiction. Therefore $P \cap Q=\{1\}$ for any Sylow $p$-subgroup $Q$ of $G$ distinct from $P$. The congruence

$$
\left|G: N_{G}(P)\right| \equiv 1\left[p^{n}\right]
$$

now follows by a well-known refinement of Sylow's Theorem (see [5], 6.5.3, p.147). If $\lambda$ were equal to 0 , then $G$ would equal $N_{G}(P)$ and hence be solvable, an absurdity.

Lemma 3.9. $\left|N_{0}\right|=\left|H: H_{0}\right|$.

Proof. $\left|N_{0}\right|$ divides

$$
\begin{aligned}
|G| & =|P||H| \\
& =|P|\left|H_{0}\right|\left|H: H_{0}\right| \\
& =\left|\left(N_{G}(P)\right)_{0}\right|\left|H_{0}\right|\left|H: H_{0}\right| .
\end{aligned}
$$


By Proposition 2.4 and Lemma 3.5, $\left|N_{0}\right|$ is prime to $\left|\left(N_{G}(P)\right)_{0}\right|$ and to $\left|H_{0}\right|$, therefore it divides $\left|H: H_{0}\right|$.

Conversely $\left|H: H_{0}\right|=\omega\left(h H_{0}\right)$ divides $\omega(h)=|<h>|$, that divides $\left|N_{0}\right| ;$ thus $\left|H: H_{0}\right|=\left|N_{0}\right|$.

Let us write $k=\left|H_{0}\right|, a=\left|N_{0}\right|=\left|\frac{H}{H_{0}}\right|=\omega\left(h H_{0}\right), \delta=\left|N_{G}(P): P\right|=$ $\left|N_{H}(P)\right|, \alpha=\left|N: N_{0}\right|$; by (5), $\alpha \geq 2$ and $\delta \geq 2$. Corollary 3.7 gives us:

$$
\begin{aligned}
p^{n} k a & \leq 1+\left(1+\lambda p^{n}\right)\left(p^{n}-1\right)+p^{n}(k-1)+\frac{p^{n} k}{\alpha}(a-1) \\
& =p^{n}\left(1+\lambda\left(p^{n}-1\right)+k-1+\frac{k}{\alpha}(a-1)\right)
\end{aligned}
$$

i.e. :

$$
k a\left(1-\frac{1}{\alpha}\right) \leq k+\lambda\left(p^{n}-1\right)-\frac{k}{\alpha}
$$

whence :

$$
k(a-1)\left(1-\frac{1}{\alpha}\right) \leq \lambda\left(p^{n}-1\right) .
$$

But

$$
1+\lambda p^{n}=\left|G: N_{G}(P)\right|=\frac{p^{n} k a}{p^{n} \delta}=\frac{k a}{\delta},
$$

thus :

$$
(* *) \quad \frac{k a}{\delta}-k(a-1)\left(1-\frac{1}{\alpha}\right) \geq 1+\lambda p^{n}-\lambda\left(p^{n}-1\right)=1+\lambda \geq 2 .
$$

LEMMA 3.10. $\delta=2$.

Proof. If $\delta \geq 3$ then $(* *)$ yields:

$$
\frac{k a}{3}-k(a-1)\left(1-\frac{1}{\alpha}\right) \geq 2,
$$

whence :

$$
\begin{gathered}
\frac{k a}{3}-k\left(\frac{a-1}{2}\right) \geq 2, \text { i.e. }: \\
\frac{k}{6}(3-a) \geq 2,
\end{gathered}
$$


whence $a<3$. But then $a=2$ and $\left|N_{0}\right|=2$. Let $N_{0}=\{1, y\}$; it follows from Lemma 1.3 that :

$$
N=N_{G}\left(N_{0}\right) \subseteq C_{G}(y) \subseteq N_{0},
$$

whence $N=N_{0}$, contradicting (5).

LEMMA 3.11. $\alpha=2$.

Proof. If $\alpha \geq 3$, then:

$$
\begin{aligned}
\frac{k a}{2} & =\frac{k a}{\delta} \\
& \geq 2+k(a-1)\left(1-\frac{1}{\alpha}\right) \\
& \geq 2+k(a-1)\left(1-\frac{1}{3}\right) \\
& >\frac{2}{3} k(a-1),
\end{aligned}
$$

whence :

$$
4(a-1)<3 a,
$$

i.e. :

$$
a<4,
$$

that is:

$$
a \in\{1,2,3\} .
$$

But then $\left|N_{0}\right| \leq 3$; let $N_{0}=<y>$. Again $C_{G}\left(N_{0}\right)=C_{G}(y) \subseteq N_{0}$, and:

$$
\alpha=\left|N: N_{0}\right| \leq\left|N_{G}\left(N_{0}\right): C_{G}\left(N_{0}\right)\right| \leq\left|A u t\left(N_{0}\right)\right| \leq 2,
$$

a contradiction. Therefore $\alpha=2$.

Proposition 3.12. If $\left(M, M^{\prime}\right) \in \mathcal{M S}(G)^{2}$ and $M_{0}^{\sharp} \cap M_{0}^{\prime \sharp} \neq \emptyset$, then $M=M^{\prime}$.

Proof. From $M_{0} \cap M_{0}^{\prime} \neq\{1\}$ follows $\left(\left|M_{0}\right|,\left|M_{0}^{\prime}\right|\right) \neq 1$, therefore Proposition 2.4 implies that $M$ and $M^{\prime}$ are conjugate. Let $M^{\prime}=M^{x}$; as $M_{0}^{\sharp} \neq \emptyset$ and $M_{0}^{\prime \sharp} \neq \emptyset, M_{0}^{\prime}=M_{0}^{x}$ by Lemma 2.5. Then

$$
M_{0} \cap M_{0}^{x}=M_{0} \cap M_{0}^{\prime} \neq\{1\}
$$

whence $x \in M$ by (3) and $M^{\prime}=M^{x}=M$. 
LEMMA 3.13.

$$
|G|=1+\left|G: N_{G}(P)\right|(|P|-1)+|G: H|\left(\left|H_{0}\right|-1\right)+|G: N|\left(\left|N_{0}\right|-1\right) \text {. }
$$

Proof. One applies the same reasoning as for Corollary 3.7, using Proposition 3.12 and (4).

Proposition 3.14. $\frac{k}{2}=1+\lambda, p$ is odd and $p^{n}-a$ divides $p^{n}-1$.

Proof. By Lemma 3.10, $\delta=2$, whence

$$
1+\lambda p^{n}=\frac{k a}{\delta}=\frac{k a}{2} .
$$

Lemma 3.13 now gives, by using the equality $\alpha=2$ (Lemma 3.11):

$$
p^{n} k a=1+\frac{k a}{2}\left(p^{n}-1\right)+p^{n}(k-1)+\frac{1}{2} p^{n} k(a-1)
$$

i.e.:

or

$$
0=1-\frac{k a}{2}+p^{n}(k-1)-\frac{1}{2} p^{n} k
$$

$(* * *)$

$$
\frac{k}{2}\left(p^{n}-a\right)=p^{n}-1
$$

Thus :

$$
\frac{k}{2} p^{n}-\frac{k a}{2}=p^{n}-1
$$

As

one has :

$$
1+\lambda p^{n}=\frac{k a}{2}
$$

$$
\begin{aligned}
(1+\lambda) p^{n} & =p^{n}-1+1+\lambda p^{n} \\
& =\frac{k}{2} p^{n}-\frac{k a}{2}+\frac{k a}{2} \\
& =\frac{k}{2} p^{n}
\end{aligned}
$$

i.e. :

$$
\frac{k}{2}=1+\lambda
$$

in particular, $k$ is even, therefore $p \neq 2$ because $p \nmid k$. (***) now becomes:

$$
p^{n}-1=(1+\lambda)\left(p^{n}-a\right),
$$

whence $p^{n}-a \mid p^{n}-1$. 
Corollary 3.15. $a=p^{n}-2$ and $k=p^{n}-1$.

Proof. $N$ acts on the set $\Omega$ of the conjugates of $N_{0}$. If $N_{0}^{x} \in \Omega$ and $N_{0} \cap N_{G}\left(N_{0}^{x}\right) \neq\{1\}$, then (cf. Lemma 2.5) $N_{0} \cap N^{x} \neq\{1\}$. But $N_{0}$ is a Hall subgroup of $N$ (Corollary 1.4), whence $N_{0} \cap N_{0}^{x} \neq\{1\}$; therefore (by (3)) $x \in N$ and $N_{0}^{x}=N_{0}$.

Any orbit of $N_{0}$ on $\Omega$, other than $\left\{N_{0}\right\}$, has therefore length $\left|N_{0}\right|$, whence

$$
|\Omega| \equiv 1\left[\left|N_{0}\right|\right]
$$

that is :

$$
|G: N| \equiv 1\left[\left|N_{0}\right|\right]
$$

(we have used the fact that

$$
\left.|\Omega|=\left|G: N_{G}\left(N_{0}\right)\right|=|G: N|\right) .
$$

Thus:

$$
a \mid \frac{p^{n} k}{\alpha}-1=\frac{p^{n} k}{2}-1=p^{n}(1+\lambda)-1 .
$$

But $p^{n}-1=(1+\lambda)\left(p^{n}-a\right)$ (see the proof of Proposition 3.14), therefore $a$ divides $1+\lambda p^{n}$, hence $a$ divides $p^{n}-2$. If $a \neq p^{n}-2$, then $a \leq \frac{1}{2}\left(p^{n}-2\right)$, that is $p^{n}-a \geq \frac{1}{2}\left(p^{n}+2\right)>\frac{1}{2}\left(p^{n}-1\right)$ and Proposition 3.14 gives $p^{n}-a=p^{n}-1$, i.e. $a=1$, a contradiction. Thus $a=p^{n}-2$; but now :

$$
\frac{k}{2}\left(p^{n}-2\right)=\frac{k a}{2}=1+\lambda p^{n}=1+\left(\frac{k}{2}-1\right) p^{n}
$$

whence $k=p^{n}-1$.

THEOREM 3.16. Under hypothesis $(\mathcal{H})$, one of the following holds:

(i) $p$ is a Fermat prime $\left(p=2^{2^{m}}+1\right)$ for some $m \geq 1$, and $G \simeq S L_{2}\left(\mathbf{F}_{2^{2}}{ }\right)$

(ii) $p=3$ and $G \simeq S L_{2}\left(\mathbf{F}_{8}\right)$.

In both cases, $H$ is the normalizer of a Sylow 2-subgroup of $G$.

Proof. $\left|H_{0}\right|=k$ is even (Proposition 3.14), therefore $H_{0}$ contains an element $t$ of order 2 ; by Lemma $1.3, C_{G}(t) \subseteq H_{0}$, therefore the number of conjugates of $t$ under $H$ is :

$$
\left|H: C_{H}(t)\right|=\left|H: C_{G}(t)\right| \geq\left|H: H_{0}\right|=a=p^{n}-2=k-1=\left|H_{0}\right|-1 .
$$


Therefore $H_{0}=\{1\} \cup\left\{t^{x} \mid x \in H\right\}$ only has elements of order 1 or 2, i.e. is a nontrivial elementary abelian 2-group; by Lemma 1.3 it is the centralizer of each of its nonidentity elements, and by Corollary 1.4 it is a Sylow 2subgroup of $G$. It follows readily that every element of $G$ has order 2 or an odd number; as in [4], p.837, one finishes the proof using [2] and the fact that $G$ is not solvable (the case of the Brauer-Suzuki-Wall that we use should actually be called Burnside's Theorem, a fact of which I was unfortunately unaware while writing [4]). The last assertion follows from Lemma 2.5: $H=N_{G}\left(H_{0}\right)$.

\section{$\S 4$. Corollaries and remarks}

Corollary 4.1. Let $G$ be a (non-abelian) simple CA-group containing a solvable Hall $p^{\prime}$-subgroup for some prime $p$ dividing its orderi,; then either $p=3$ and $G$ is isomorphic to $S L_{2}\left(\mathbf{F}_{8}\right)$, or $p$ is a Fermat prime other than 3 and $G$ is isomorphic to $S L_{2}\left(\mathbf{F}_{p-1}\right)$.

Remark. This is the main Theorem of [4].

Proof. By Proposition 2.6, $G$ satisfies hypothesis $(\mathcal{H})$, and one may therefore apply Theorem 3.16.

The original motivation for this paper was:

Corollary 4.2. If $G$ is a minimal counterexample to the FeitThompson Theorem that satisfies the conditions listed on p.ix of [1], then there is no prime $p \in \pi(G)$ such that $G$ possess a $p^{\prime}$-Hall subgroup.

Proof. Our conditions (1) to (5) clearly follow from the conditions listed on p.ix of [1] ; if $G$ would have a Hall $p^{\prime}$-subgroup $H$, then $H$ would be solvable(by the minimality of $G$ ), and hypothesis $(\mathcal{H}$ ) would be satisfied : Theorem 3.16 would apply. But all the groups that appear in the conclusion of this Theorem have even order.

\section{REFERENCES}

[1] H.Bender and G.Glauberman, Local analysis for the Odd Order Theorem, Cambridge University Press, Cambridge, 1994.

[2] W. Burnside, On a class of groups of finite order, Trans. Camb. Phil. Soc., 18 (1900), 269-276. 
[3] O. H. Kegel, Produkte nilpotenter Gruppen, Arch. Math., 12 (1961), 90-93.

[4] P. Lescot, A note on CA-groups, Communications in Algebra, 18 (1990), no. 3, 833-838.

[5] W. R. Scott, Group Theory, Dover, New York, 1987.

[6] J. G. Thompson, Letter (August 31st, 1990).

[7] L. Weisner, Groups in which the normalizer of every element except identity is abelian, Bull. Amer. Math. Soc., 33 (1925), 413-416.

INSSET, Université de Picardie

48 Rue Raspail

02100 Saint-Quentin

France

paul.lescot@insset.u-picardie.fr 